\title{
A RING-IMAGING DETECTOR WITH LIQUID AND SOLID RADIATORS USING A MULTISTEP PARALLEL-PLATE AVALANCHE CHAMBER AT ATMOSPHERIC PRESSURE WITH OPTICAL READOUT
}

\author{
Y. Giomataris, A. Gougas, W. Dominik*), G. Charpak, F. Sauli and N. Zaganidis
}

CERN, Geneva, Switzerland

\begin{abstract}
A parallel-plate multistep chamber, filled with $\mathrm{He}(97 \%)$ and $\mathrm{C}_{2} \mathrm{H}_{6}(3 \%)$ at atmospheric pressure, and tetrakis(dimethylamine)ethylene, with an optical readout system was tested. Laboratory tests using an ultraviolet flash lamp show a good single-photoelectron efficiency, spatial resolution better than $220 \mu \mathrm{m}$ r.m.s., and small spot-size ( $\approx 3 \mathrm{~mm}$ FWHM) for the singlephotoelectron avalanches. Tests in a $10 \mathrm{GeV}$ particle beam confirm the good performance of the device, the high efficiency, the capability of very complex and dense position-information readout, and the suitability for imaging of high-energy gamma-rays.
\end{abstract}

Presented by W. Dominik at the

Conference on Advanced Technology and Particle Physics,

Como, 13-17 June 1988

*) On leave of absence from the Institute of Experimental Physics, Warsaw University, Poland. 


\section{INTRODUCTION}

In a recent report [1], it was suggested that the direction of high-energy $\gamma$-rays can be determined by using images of Cherenkov light emitted by electrons produced in high-energy electromagnetic showers in dense materials.

The method relies on the fact that, at the beginning of a shower development, the detection of the electrons is strongly correlated with the direction of the $\gamma$-ray initiating the shower. With a parabolic or spherical mirror at the rear end of a crystal (fig. 1), it is then possible to obtain a ring-like image in a detector filled with a photo-ionizable vapour. The shape, size, and position of the ring permit the determination of the angle of incidence of the X-ray (fig. 2).

The optical retrieval of the light emitted by avalanches developing in the uniform electric field between the gap of two mesh electrodes is ideal for the imaging of events with high multiplicity of ultraviolet (UV) photons. Single photoelectrons can easily be detected with a multistep chamber filled with a gas mixture containing photosensitive compounds [2], e.g. triethylamine (TEA) or tetrakis(dimethylamine)ethylene (TMAE) [3].

These compounds play a double role in the visualization of UV photons. They allow the efficient conversion of photons to electrons and emit light during the charge multiplication process in the gas. The results of the investigation of the mechanism of light emission in the avalanche chambers are described in previous articles $[4,5]$. Operation of the detector at atmospheric pressure is very essential: it permits the construction of devices with large active areas while, at the same time, the avalanche size remains small, which is important for the case of large information density. However, one has to remember that one of the limitations of the single-photoelectron detection in gaseous detectors is the relatively high number of primary electrons liberated by the ionizing particles traversing the active volume. This condition limits the thickness of the sensitive gap and the number of potential candidates of gas mixtures to those that contain helium as a major component.

\section{DESCRIPTION OF THE DEVICE}

\subsection{The multistep parallel-plate avalanche chamber}

We have built a multistep avalanche chamber around an existing $27 \mathrm{~cm} \times 27 \mathrm{~cm}$ mechanical framework, which is shown schematically in fig. 3. Our chamber is composed of a quartz window, $16 \mathrm{~cm}$ in diameter and $5 \mathrm{~mm}$ thick, which is transparent to UV light of wavelength $>160 \mathrm{~nm}$, and a series of wire meshes. These meshes are constructed from $50 \mu \mathrm{m}$ diameter stainless-steel wires, spaced at $500 \mu \mathrm{m}$ intervals and interwoven in orthogonal directions. The first gap is a $7 \mathrm{~mm}$ low electric field region, which is the conversion gap for UV photons. Electrons produced in this region are moving perpendicularly to the mesh planes into a region of higher electric field, the preamplification region, $4 \mathrm{~mm}$ thick, where the field is sufficiently high for the entering electrons to ionize the gas mixture. Electrons are amplified by the avalanche process in this region and gains of $10^{3}$ to $10^{4}$ are attainable. As the electrons drift to the end of the preamplification stage they are partly transferred through a $51 \mathrm{~mm}$ thick uniform electric field to the amplification region, which is a $7 \mathrm{~mm}$ gap of high electric field. Total gains of $10^{5}-10^{6}$ are easily achieved. We choose for our detector a gas mixture of He and $\mathrm{C}_{2} \mathrm{H}_{6}$ at atmospheric pressure, and TMAE. The total number of electrons, produced by minimum ionizing particles in this gas mixture, is small $\left(\approx 8 \mathrm{~cm}^{-1}\right)$ and the avalanche size is expected to be smaller than the one observed in low-pressure multistep chambers ( $\sim 5.8 \mathrm{~mm}$ FWHM), which have been proved to be efficient imaging detectors for single photoelectrons [6]. At the last multiplying gap, photons are emitted isotropically during the avalanche development [4], in a number approaching the charge gain. The gas is confined with a glass (pyrex) window, which is located $10 \mathrm{~mm}$ from the last mesh plane and permits the imaging of this light with a charge-coupled device (CCD) camera. 
One very important parameter for operation of a gaseous detector containing TMAE is the concentration: it allows the achievement of full absorption of the photons in the conversion space, with small depth of this gap (fast detector and minimal parallax error), reduces the avalanche spread because of UV photon propagation during charge multiplication, allows the avoidance of photon feedback and, what is more important in the case of optical read-out, improves the light yield for a given charge gain of the detector. Therefore, the chamber was operated with a TMAE concentration corresponding to its partial vapour pressure of $1.29 \mathrm{mbar}$ and $2.46 \mathrm{mbar}$, between $35^{\circ} \mathrm{C}$ and $45^{\circ} \mathrm{C}$ respectively [7]. The TMAE photoabsorption cross-section is $\sim 30 \mathrm{Mb}[7,8]$.

In order to increase charge gain and light output we pulsed the last amplification stage of the chamber [4]; we applied a pulse of $400 \mathrm{~V}$ and $1 \mu$ s duration to the cathode of this gap. Timing was chosen in order to increase electric field when a cloud of preamplified charge entered this gap. Thus, the chamber was fully activated for a short time, working at a lower gain the rest of the time. With such a method of operation we maximized the light output without sparks.

\subsection{The optical readout system}

A CCD camera with an image-intensification system was used for the detection of the light emitted by the avalanches in the chamber. In fig. 4 is shown a schematic of the optical readout system.

The light spectrum emitted by the avalanches in a parallel-plate chamber containing TMAE, in the gas mixture, is peaked at $\approx 480 \mathrm{~nm}$ [4]. At this wavelength, even ordinary glass is highly transparent. This allows the use of pyrex for the chamber's exit window and a standard objective for the image intensifier. A Leitz Noctilux 1:1/50 mm objective, with a large aperture and good optical quality, was used.

A gateable multichannel plate image intensifier (constructed by Delft Electroniche Producten type DEP XX 1450M) was used for light multiplication (fig. 4). It has an $18 \mathrm{~mm}$ active diameter with an S20 photocathode and a P47 phosphor screen. It can operate either in continuous or gated mode with a minimal time of gate opening at full efficiency as low as 100 ns. Both photocathode and phosphor screen were deposited on the fibre-optics windows. The exit window of this first stage is coupled to the fibre optics entrance window of the second electrostatic image intensifier/reducer type DEP XX 1490, thus demagnifying the image of $18 \mathrm{~mm}$ in diameter to $7 \mathrm{~mm}$ in diameter, which therefore matches the CCD size. The gain of this second stage is 7 photons per incident photon at the nominal operating voltage of $15 \mathrm{kV}$. The demagnifier is equipped with an $\mathrm{S} 20$ photocathode and a P46 phosphor screen of $100 \mathrm{~ns}$ decay-time, allowing efficient coupling to the $\mathrm{CCD}$. The total light gain is $\approx 10^{3} \mathrm{~W} / \mathrm{W}$. We used a CCD Thomson TH 7852 FO camera with physical dimensions of $4.32 \mathrm{~mm} \times 5.82 \mathrm{~mm}$, containing $144 \times 208$ pixels. Images in standard video signal were digitized by a Data Translation DT-2851 frame-grabber connected to an IBM PC/AT computer. The selected events were read, then compacted, and written onto disk.

\section{POSITION RESOLUTION AND AVALANCHE SIZE}

We performed measurements for the position resolution and the single-avalanche size using a pulsed hydrogen lamp, with a spark gap of $1 \mathrm{~mm}$. A collimator of $200 \mu \mathrm{m}$ diameter was placed in front of the entrance of the window at $20 \mathrm{~cm}$ distance from the lamp. The intensity was attenuated by a set of Aclar $22 \mathrm{~A}$ foils in front of the lamp. The chamber's coincidence rate at full efficiency, measured by the total number of charge signals in comparison with the picked-up signals from the lamp, was less than $20 \%$, thus confirming that we were detecting either one or no photon. Figure 5 shows the light-intensity distribution from single avalanches produced in the chamber and then detected by the intensified CCD camera. The distribution has a peaked shape, proving that we obtained good efficiency for single-photoclectron detection. 
The size of single-avalanche spots is of the order of $3.0 \mathrm{~mm}$ (FWHM) and the spatial resolution of the chamber is $220 \mu \mathrm{m}$ (fig. 6). Spatial resolution was calculated taking the centroids of the spot intensity distribution. However, if we calculate it using only the pixel of maximum intensity, the spatial resolution is of the order of $100 \mu \mathrm{m}$. At present, we do not explain why the second method provides a better resolution, which in any case cannot be worse than $220 \mu \mathrm{m}$. We suspect that the difference is due to an asymmetric avalanch growth, manifesting itself as a larger dispersion in the centre of gravity.

Using the same experimental set-up we performed measurements of the drift velocity as a function of the applied electric field in the transfer region. For our standard gas mixture $-\mathrm{He}(97 \%)$, $\mathrm{C}_{2} \mathrm{H}_{6}(3 \%)$ - and TMAE at $40{ }^{\circ} \mathrm{C}$, we observed a plateau for the electric field above a value of $200 \mathrm{~V} / \mathrm{cm}$ (fig. 7). This allowed a relatively low electric field, $600 \mathrm{~V} / \mathrm{cm}$, to be applied to the transfer region, thus reducing the maximum voltages needed for the whole chain of meshes. However, the electric field cannot be too low, because the transfer efficiency would degrade. Charge transfer efficiency depends on the field ratio in these two regions and a value of $10 \%$ is reasonable. The possibility of having a constant delay owing to the transfer time is one of the main advantages of the multistep avalanche chamber. It permits the chamber's gating by an external trigger and the operation of the detector in a high-rate environment [6].

\section{TESTS USING A CHARGED-PARTICLE BEAM}

\subsection{Results with a $\mathrm{BaF}_{2}$ radiator}

A radiator with a spherical mirror on the back surface of a $5 \mathrm{~cm}$ thick (2.44 radiation lengths) $\mathrm{BaF}_{2}$ crystal placed against the first mesh plane of conversion gap, was tested. The radius of curvature of the reflecting surface was $16 \mathrm{~cm}$. The size of the crystal, which was $12 \mathrm{~cm}$ wide, was unfortunately slightly too small for full acceptance of the reflected light. An electron or a $\gamma$-ray hitting the centre of the crystal would give a ring with a radius of $6 \mathrm{~cm}$. With an incoming angle of $30^{\circ}$ Monte Carlo calculations showed that an arc of a circular-like image should be visible (fig. 2). A preliminary test was made with the same photon detector as the one used for the spot-size and spatial-resolution measurements described above.

It became apparent that the main obstacle to a correct measurement was the detection of the scintillation light in the crystal. $\mathrm{BaF}_{2}$ is known to emit a broad spectrum of $\mathrm{UV}$ photons, containing two components [9] - one fast, peaked at $195 \mathrm{~nm}$ and $220 \mathrm{~nm}$ to which TMAE vapours are sensitive [10], and a slow component at around $300 \mathrm{~nm}$. The intensity is so large that at a chamber charge gain appropriate for single-photon detection, the image intensifier saturates. Working with a low gain permitted one to see (as expected) a ring of small intensity, at the edge of the crystal (fig. 8). However, such a ring could also have been interpreted as the reflection of the scintillation light by the metallic structures holding the crystal at the back. The intense spot clearly corresponds to the shower development: the scintillation light is isotropic, but most of it is internally reflected (total reflection angle $\approx 50^{\circ}$ ).

We have repeated this experiment with some changes: we blackened all the metallic structures holding the crystal; we put an opaque plastic sheet on one half of the crystal, onto which we were sending the incident radiation; and we varied the angles of incidence. As shown in fig. 9, the situation was far from ideal since the scintillation component still gives a fake ring overlapping the Cherenkov ring, due to reflections on the unpolished sides. We performed the test detecting photons emitted in the crystal directly, having removed the chamber, and we detected only the visible component of the spectrum with the image intensifier. Figure 10 shows the Cherenkov ring detected between the scintillation spot and the reflections in the edges. This image is obtained by overlapping images produced by $\approx 10^{3}$ pions. It is clear that the strong scintillation light (estimated to be 4 times more intense than the Cherenkov light) as well as the large index of refraction of $\mathrm{BaF}_{2}$, causing the loss of 
signal, are obstacles for our purposes. We decided to replace the $\mathrm{BaF}_{2}$ crystal by another non-scintillating radiator with smaller refractive index.

\subsection{Liquid-freon radiator}

A second prototype, with a liquid radiator, was constructed (fig. 11). It consists of the same parallel-plate multistep chamber coupled to a liquid radiator. The radiator is a stainless-steel cylinder $15 \mathrm{~cm}$ in diameter and $7 \mathrm{~cm}$ in length. The quartz window of the first prototype, mentioned above, was replaced by a $\mathrm{CaF}_{2}$ window $5 \mathrm{~mm}$ thick and $14 \mathrm{~cm}$ in diameter. The radiator was placed in front of the $\mathrm{CaF}_{2}$ window, since it is obvious that the effective cut-off around $170 \mathrm{~nm}$ was due to the freon transmission [11] as in the case of the quartz window. A spherical mirror facing the chamber was positioned in order to focus on the window the Cherenkov photons produced by charged particles inside the radiator. The mirror's radius of curvature was $13 \mathrm{~cm}$ and its diameter $16 \mathrm{~cm}$; it was held by a metallic support at a distance of $5.2 \mathrm{~cm}$ from the chamber's window. In a following paper it will be shown that the focalized $\mathrm{RICH}$ has some advantages over the proximity-focusing $\mathrm{RICH}$ detector [8]: negligible geometric error, lower refraction shift on the window, and economy (one window is needed instead of two).

The liquid chosen is a freon compound $\left(\mathrm{C}_{6} \mathrm{~F}_{14}\right)$, which circulates in a closed circuit and is purified through an Oxisorb filter cartridge. The liquid-handling system was heated to the temperature of the chamber to avoid condensation of TMAE on the window.

The apparatus was tested in a $10 \mathrm{GeV}$ unseparated beam containing mostly pions in order to demonstrate the ring-imaging capabilities. Triggers were obtained from three scintillating counters $\left(\mathrm{H}_{1}, \mathrm{H}_{2}, \mathrm{H}_{3}\right)$, placed in front of the detector, and another one $\left(\mathrm{H}_{4}\right)$ behind the apparatus. The fine tracking of the particles was defined mainly by the $\mathrm{H}_{2}$ and $\mathrm{H}_{3}$ counters, which were covering a $5 \mathrm{~mm} \times 5 \mathrm{~mm}$ surface. The final trigger was given by the coincidence signal of 4 counters and a threshold Cherenkov counter (allowing the selection of hadrons and electrons), which controlled the gating of chamber, the CCD camera, and the data-acquisition system.

The results demonstrate the good performance of the detector. Figure 12 shows a typical Cherenkov ring corresponding to pions to $10 \mathrm{GeV} / \mathrm{c}$ momentum passing through the radiator. In order to suppress the spots induced by single photons in the conversion gap a threshold was applied on the spot intensity, according to the distribution of fig. 7 . The average number of points on the ring is around 20, as is expected by the given TMAE temperature and the transparency of the first mesh. Owing to the experimental set-up, particles were traversing the chamber, ionizing the gas mixture inside. In the case of a gas mixture containing mainly $\mathrm{He}$ the charge liberated by minimum ionizing particles in the thin conversion gap is of the order of five electrons, giving a spot comparable to the single-photoelectron spot. Therefore, one can detect charged particles [spot inside the ring (fig. 12)] together with UV photoelectrons without strong limitation due to ionization.

Selecting electrons at $10 \mathrm{GeV} / \mathrm{c}$, we detected Cherenkov rings corresponding to electromagnetic showers. Because the liquid radiator is not very dense ( 0.3 radiation length including the thickness of the $\mathrm{CaF}_{2}$ window) we installed an optically transparent lead-glass plate $1 \mathrm{~cm}$ thick (1 radiation length) on the pyrex entrance window of the chamber. An example of the image obtained by the electromagnetic shower development is shown in fig. 13. The ring shape is similar to that of a single charged particle, but it contains many more points because of the higher multiplicity of the electromagnetic shower.

Pulsing the last amplification gap appeared to be a very powerful technique for single-photoelectron detection. Applying a $400 \mathrm{~V}$ pulse we activated this stage for a short time corresponding to the spread in time of our source (drift-time in the conversion gap), therefore avoiding sparking (operating still in the proportional region). It is known that gating the charge transfer to the last stage permits fast event selection [12] and increases the total chamber gain in a 
high particle flux environment. Gating is performed by introducing a plane made of wires of opposite polarity on the adjacent wires. In the next version of the detector we are going to combine gating with pulsing techniques. This is an advantage of the optical readout of information from gaseous detectors compared to electronic readout, since the problem of high-voltage pulse pick-up by sensitive electronis is avoided - which, in the case of charge readout, may kill the signal.

The parallel-plate chamber at normal pressure allows the achievement for a given charge gain, of a higher light output from the avalanches than that of the low-pressure chambers [5], which is very important for optical readout.

\section{CONCLUSIONS AND DISCUSSION}

These first experimental results confirm the high single-photoelectron efficiency of our detector and demonstrate the possibility of using atmospheric-pressure multistep avalanche chambers with optical read-out for single-photoelectron detection. Moreover, the high spatial resolution, the small spotsize, the weak intensity of primary ionization, and the absence of feedback photoelectrons, makes such a device attractive when used for Ring Imaging Cherenkov ( $\mathrm{RICH}$ ) techniques and the imaging of electromagnetic showers.

One of the main obstacles for operation in gaseous detectors containing TMAE is the propagation of UV photons emitted during charge multiplication [13]. A multistep parallel-plate chamber with a wide transfer gap is a favourable structure allowing the avoidance of this source of gain limitation and of pattern-recognition ambiguities. However, the TMAE concentration should be high and the first stage should be operated at moderate charge gain. As we can observe in our typical image (fig. 12), we did not suffer from feedback photoelectrons.

In a following paper, we are going to give a final off-line analysis of the results concerning the quality factor of our RICH detector and its capabilities in charged-particle identification. Furthermore, the angular resolution for electromagnetic shower imaging is currently under study.

\section{Acknowledgements}

We have had a lot of very competent help. For this we would like to thank R. Bouclier and J.-C. Santiard from CERN, and K. Oikonomou from the University of Thessaloniki. We are grateful to J. Séguinot, T. Ypsilantis and A. Breskin for the interest they have taken in our work and the enlightening discussions we had. M. Bosteels helped us with the construction of the liquid circulation system. We would also like to thank N. Solomey and K. Zioutas for their assistance in difficult moments. C. Nichols and A. Braem prepared the crystals we used and the radiator's mirror, all of them of remarkable quality. We want also to thank the CERN PS Operation Group, especially K. Batzner and the PS Coordinator P. Bloch. Last, but not least, F. Lemeilleur gave us valuable help with the triggering of the apparatus. 


\section{REFERENCES}

[1] Y. Giomataris and G. Charpak, paper submitted to the 20th Int. Cosmic Ray Conf., Moscow, 1987.

[2] M. Suzuki, A. Breskin, G. Charpak, E. Daubie, W. Dominik, J.-P. Fabre, J. Gaudean, F. Sauli, D. Sauvage, P. Strock and T. Zeludziewicz, Nucl. Instrum. Methods A263 (1988) 237.

[3] G. Charpak and F. Sauli, Nucl. Instrum. Methods 225 (1984) 627.

[4] G. Charpak, J.-P. Fabre, F. Sauli, M. Suzuki and W. Dominik, Nucl. Instrum Methods, A258 (1987) 177.

G. Charpak, W. Dominik, J.-P. Fabre, J. Gaudaen, F. Sauli and M. Suzuki, Nucl. Instrum. Methods, A269 (1988) 142.

[5] D. Sauvage, A. Breskin and R. Chechik, Weizmann Institute preprint WIS-88/8 (1988), submitted to Nucl. Instrum. Methods.

[6] A. Breskin, R. Chechik, Z. Fraenkel, D. Sauvage, V. Steiner, I. Tserruya, G. Charpak, W. Dominik, J.-P. Fabre, J. Gaudean, F. Sauli, M. Suzuki, P. Glässel, H. Ries, A. Schön and $\mathrm{H}$. Specht, presented at the London Conference on Position Sensitive Detectors, London, 1987.

[7] Y. Giomataris et al., internal note DELPHI 86-17, RICH-15 (1986).

D. Anderson, Fermilab preprint FN-473 (1988), submitted to Nucl. Instrum. Methods A.

[8] R. Arnold, P. Baillon, H.J. Besch, M. Bosteels, E. Christophel, M. Dracos, Y. Giomataris, J.L. Guyonnet, G. Passardi, P. Pétroff, J. Séguinot and T. Ypsilantis, Strasburg preprint CRN/HE 87-08 (1987).

R. Arnold, Y. Giomataris, J.L. Guyonnet, P. Pétroff, J. Séguinot and T. Ypsilantis, preprint CERN-EP/87-186 (1987).

[9] P. Schotanus, C. Van Eijk and R. Hollander, Nucl. Instrum. Methods A269 (1988) 377.

[10] R.A. Holroyd, J.A. Preses and C.L. Woody, Nucl. Instrum. Methods 261 (1987) 440.

S. Ekelin, Internal Report, Royal Institute of Technology, Stockholm (1981).

[11] R. Arnold, P. Baillon, J.D. Berst, H.J. Besch, M. Bosteels, E. Christophel, Y. Giomataris, J.L. Guyonnet, G. Passardi, J. Séguinot, J. Tocqueville, D. Toët and T. Ypsilantis, Nucl. Instrum. Methods, A252 (1986) 188.

J. Séguinot et al., 2nd Hellenic School on Elementary Particle Physics, 1985 (World Scientific, Singapore, 1986), p. 61-190.

[12] A. Breskin, G. Charpak, S. Majewski, G. Melchart, A. Peisert, F. Sauli, F. Mathy and G. Petersen, Nucl. Instrum. Methods 178 (1980) 11.

[13] R. Arnold, P. Baillon, H.J. Besch, M. Bosteels, E. Christophel, M. Dracos, Y. Giomataris, J.L. Guyonnet, G. Passardi, P. Pétroff, J. Séguinot and T. Ypsilantis, Strasburg preprint CRN/HE 87-01 (1987).

W. Dominik, D. Anderson, A. Breskin, G. Charpak, F. Sauli and S. Majewski, preprint CERN-EP/84-29 (1984). 


\section{Figure captions}

Fig. 1 Principle of a high-energy $\gamma$-ray Cherenkov telescope. The radiator is placed between a spherical mirror and a photosensitive gas detector.

Fig. 2 Typical rings obtained from $10 \mathrm{GeV}$ gammas in 2.5 radiation lengths of $\mathrm{BaF}_{2}$ :
a) normal incidence,
b) two gammas at $60^{\circ}$,
c) two gammas at $30^{\circ}$.

Fig. 3 Schematics of a RICH detector.

Fig. 4 Schematic view of the optical readout system.

Fig. 5 Distribution of the intensity of light emitted by single-photoelectron avalanches in the chamber.

Fig. 6 a) Size of single-photoelectron avalanches in the chamber.

b) Position resolution with optical readout. Photon beams of $0.2 \mathrm{~mm}$ diameter at a distance of $1.9 \mathrm{~mm}$; distribution of centroids of single-photoelectron avalanches.

Fig. 7 Drift velocity as a function of electric field in $\mathrm{He}(97 \%)+\mathrm{C}_{2} \mathrm{H}_{6}(3 \%)+$ TMAE at $34{ }^{\circ} \mathrm{C}$.

Fig. 8 Image of an electromagnetic shower in $\mathrm{BaF}_{2}$.

Fig. 9 Image of an electromagnetic shower in $\mathrm{BaF}_{2}$; the scintillating half of the crystal is covered by kapton foil.

Fig. 10 Direct image of $10^{3}$ pions in $\mathrm{BaF}_{2}$ (chamber removed).

Fig. 11 Schematics of the liquid radiator construction.

Fig. 12 Image of a $10 \mathrm{GeV} / \mathrm{c}$ single pion in the liquid-freon radiator.

Fig. 13 Image of a $10 \mathrm{GeV} / \mathrm{c}$ electromagnetic shower in the liquid-freon radiator (shower starts in the lead-glass plate). 

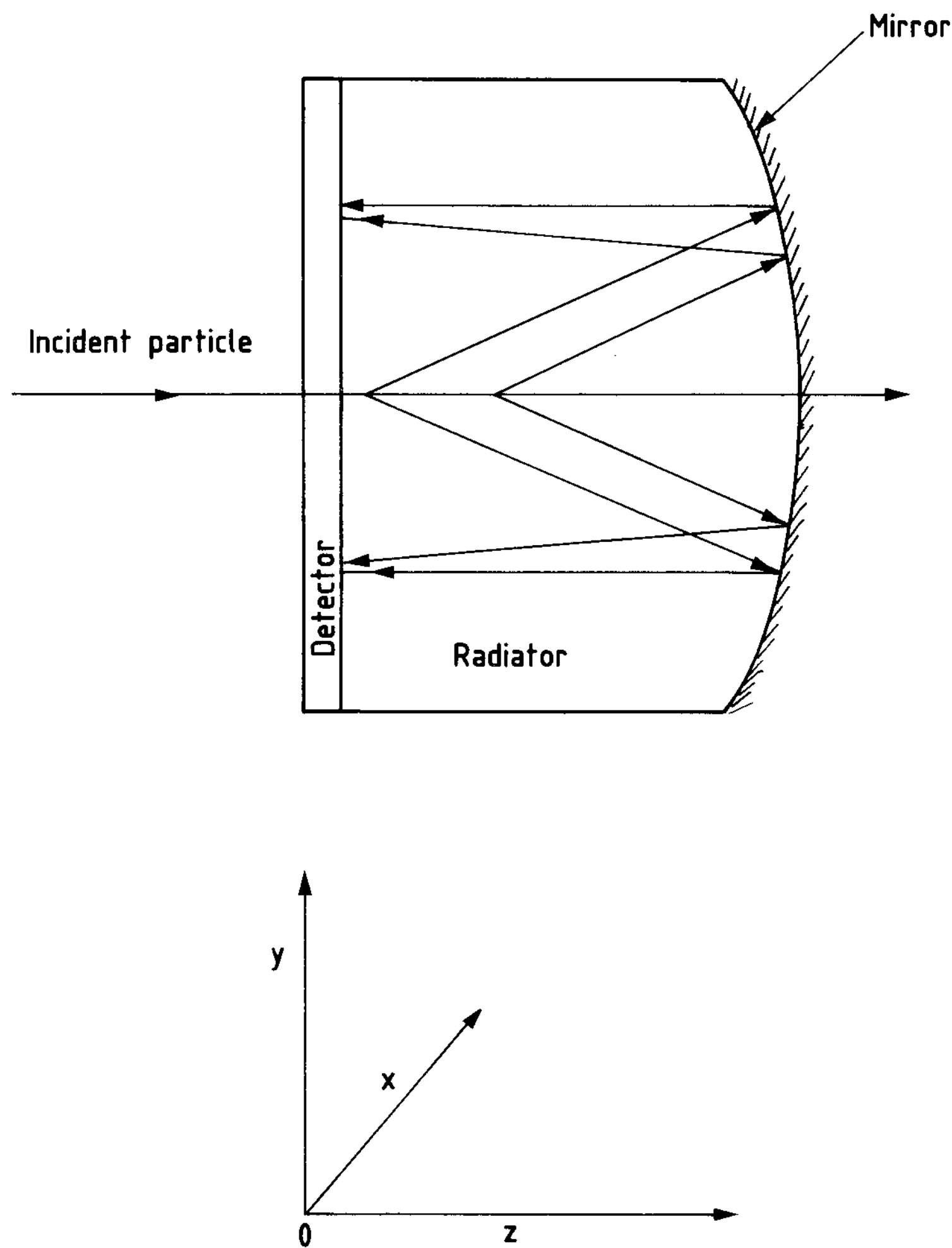

Fig. 1 

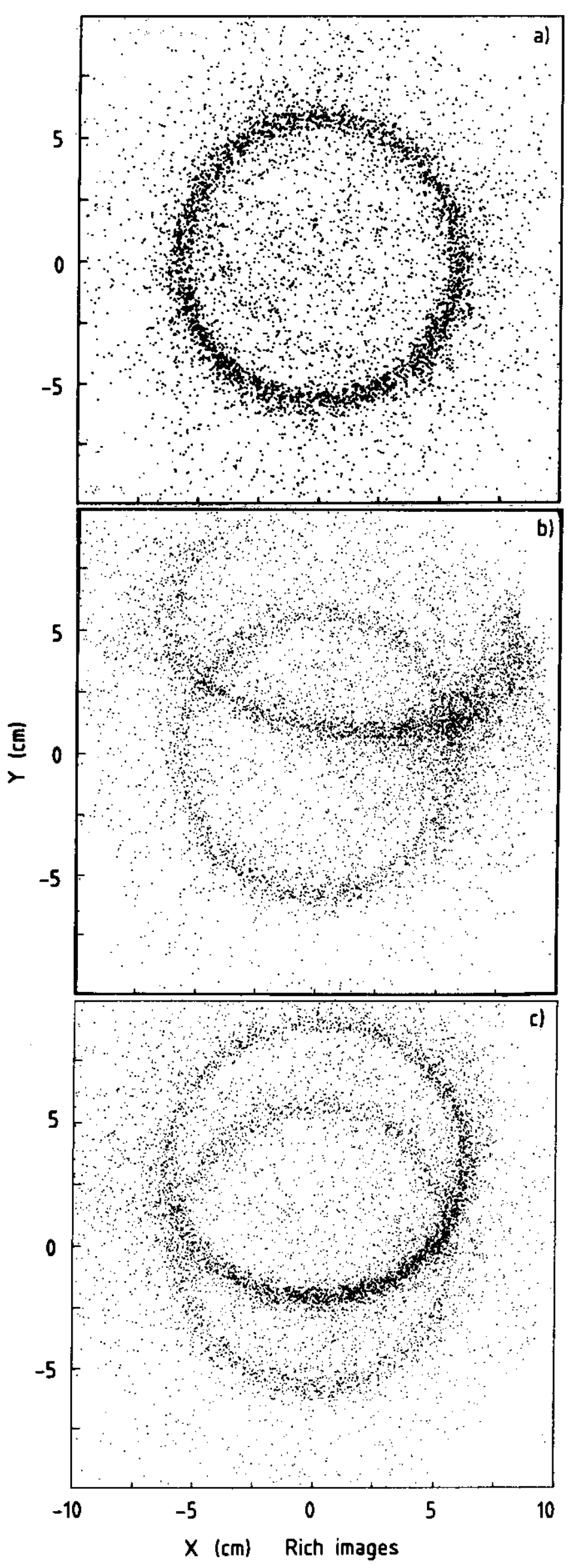

Fig. 2 


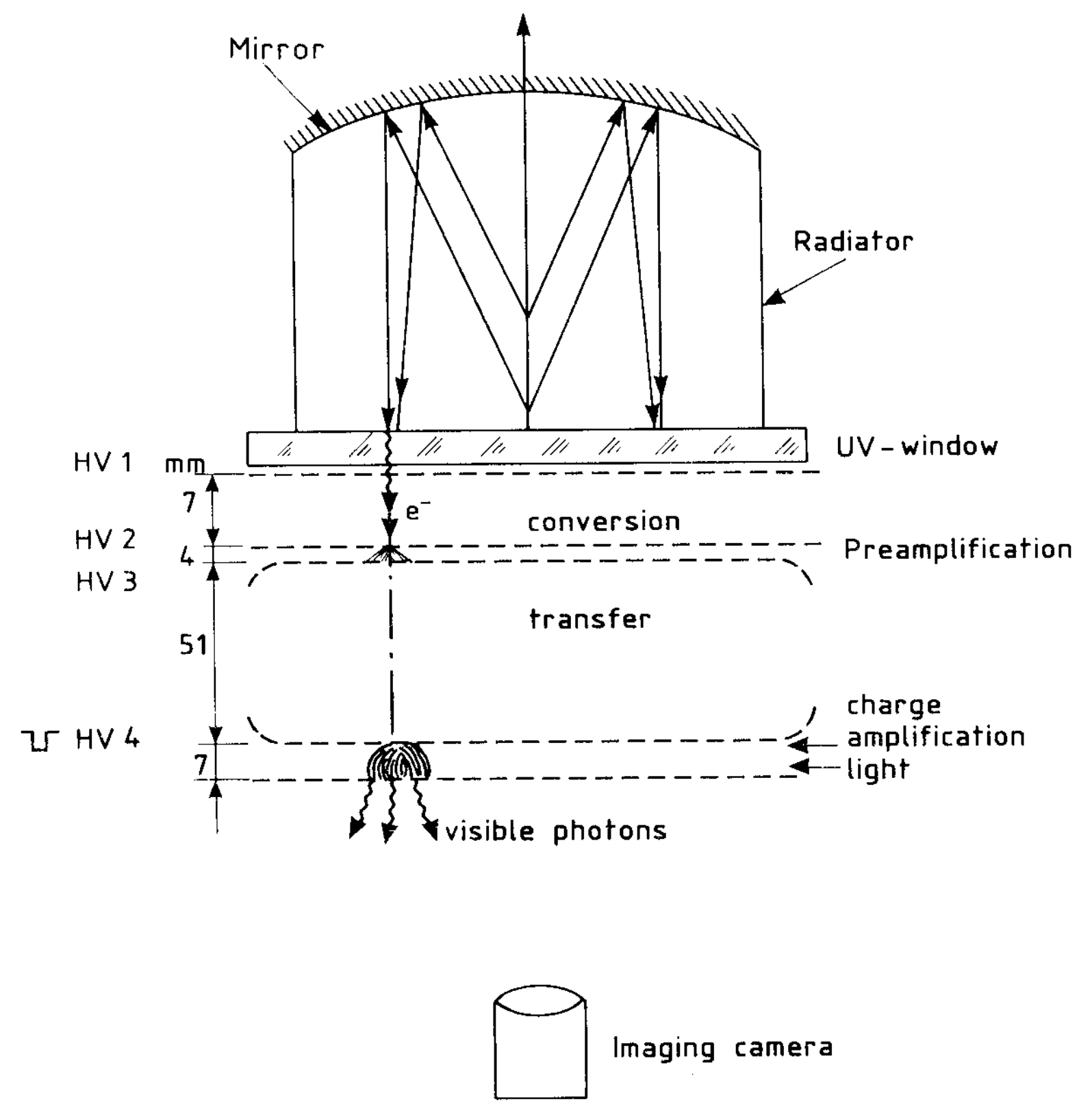

Fig. 3 


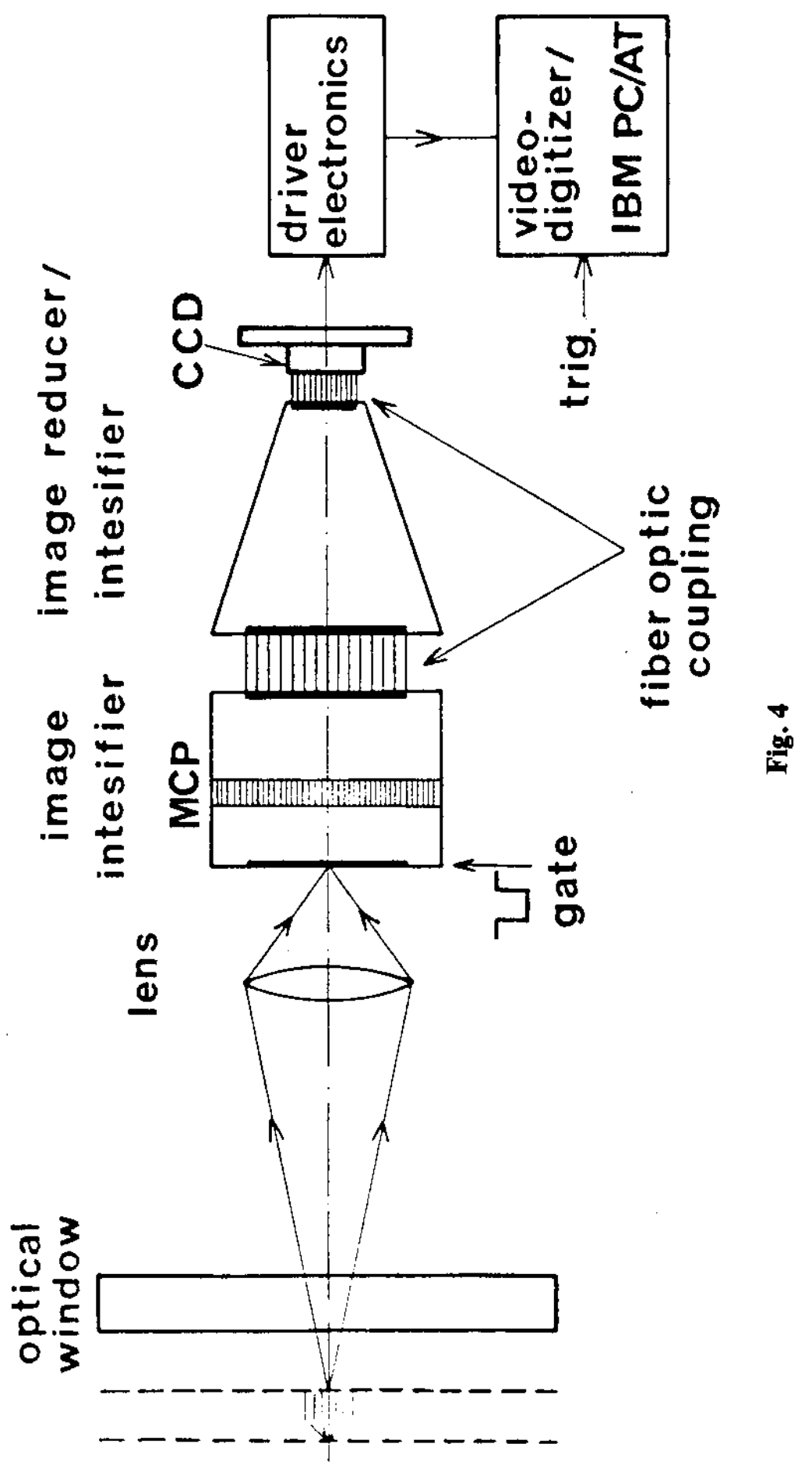




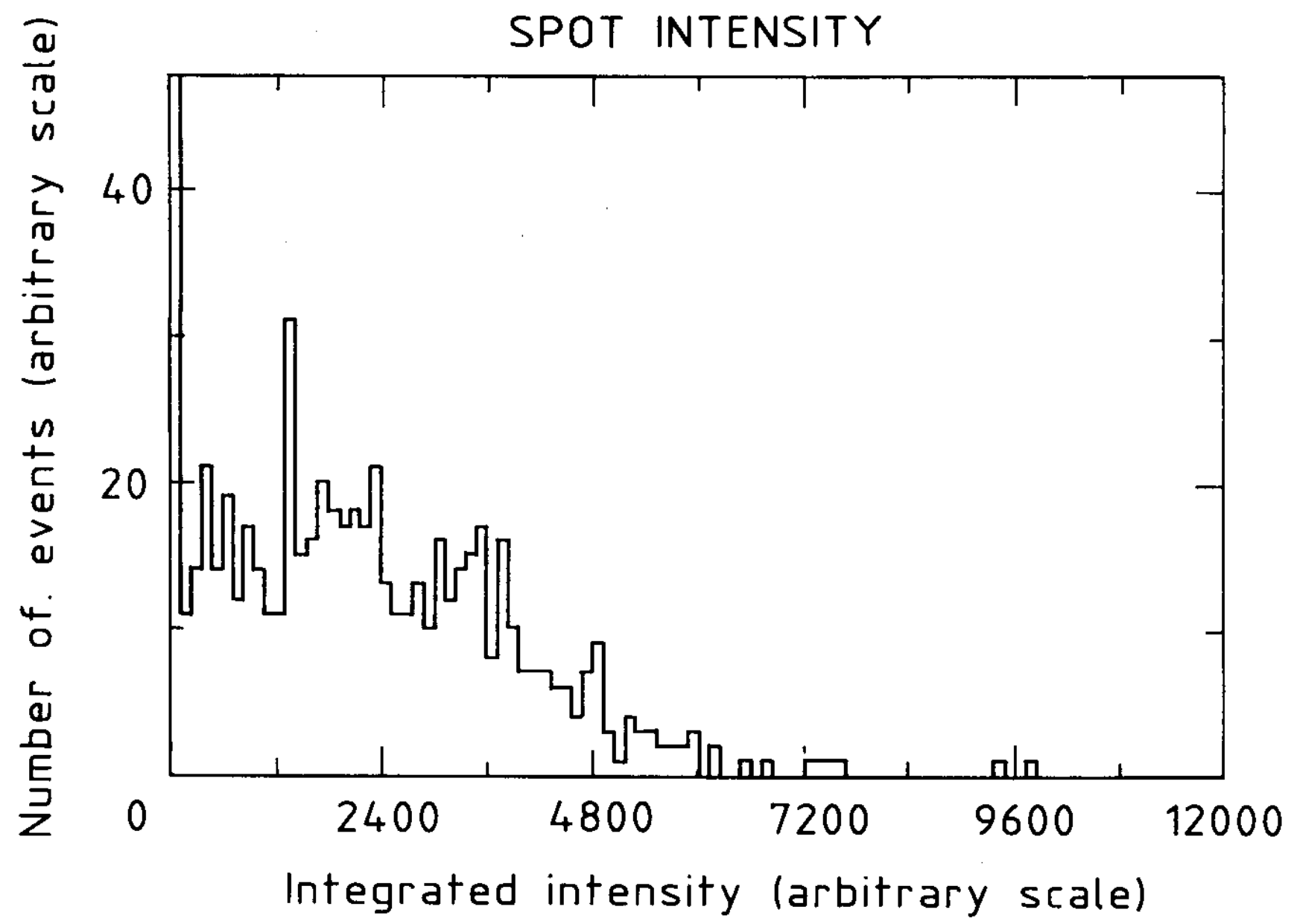

Fig. 5 

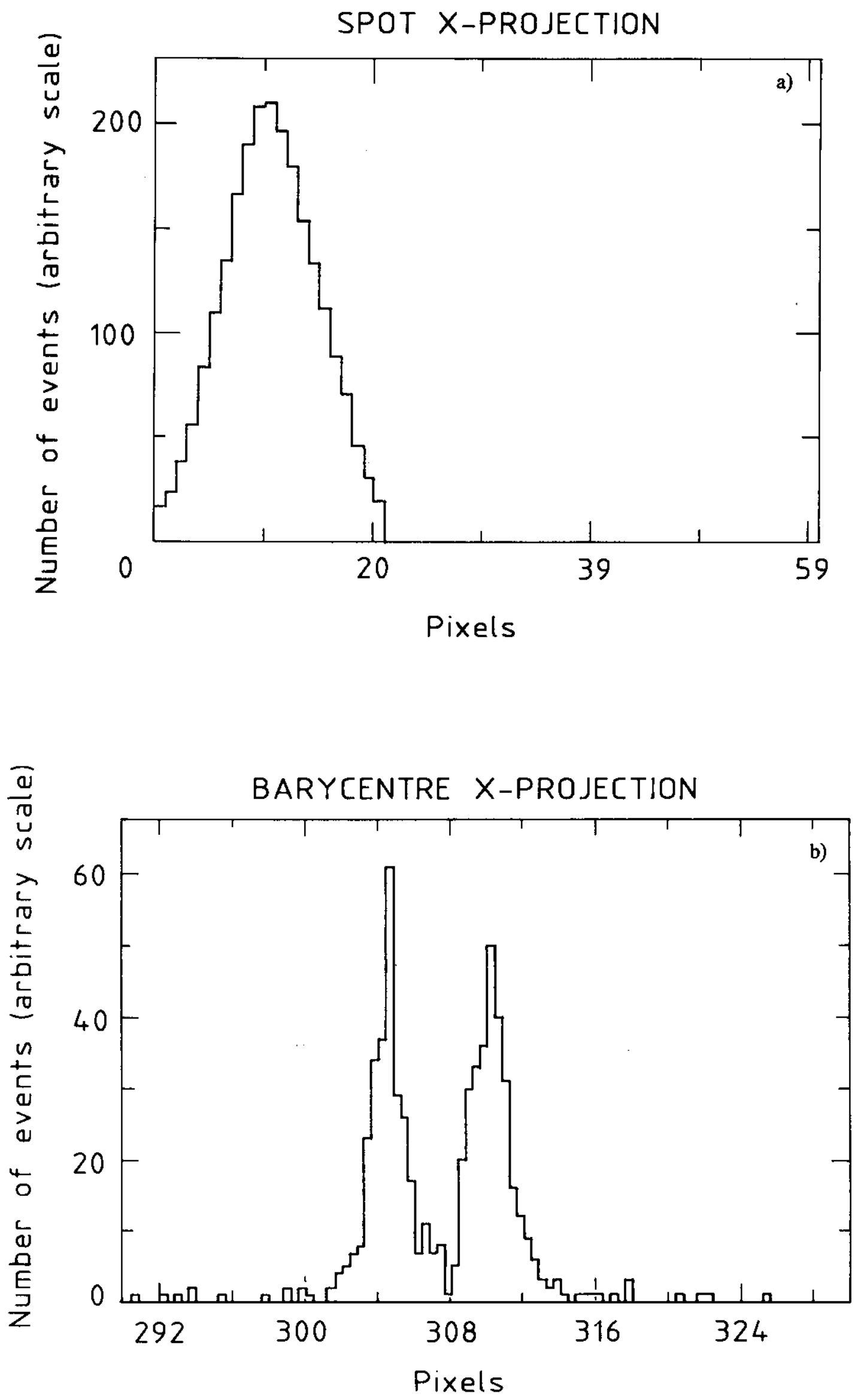

Fig. 6 


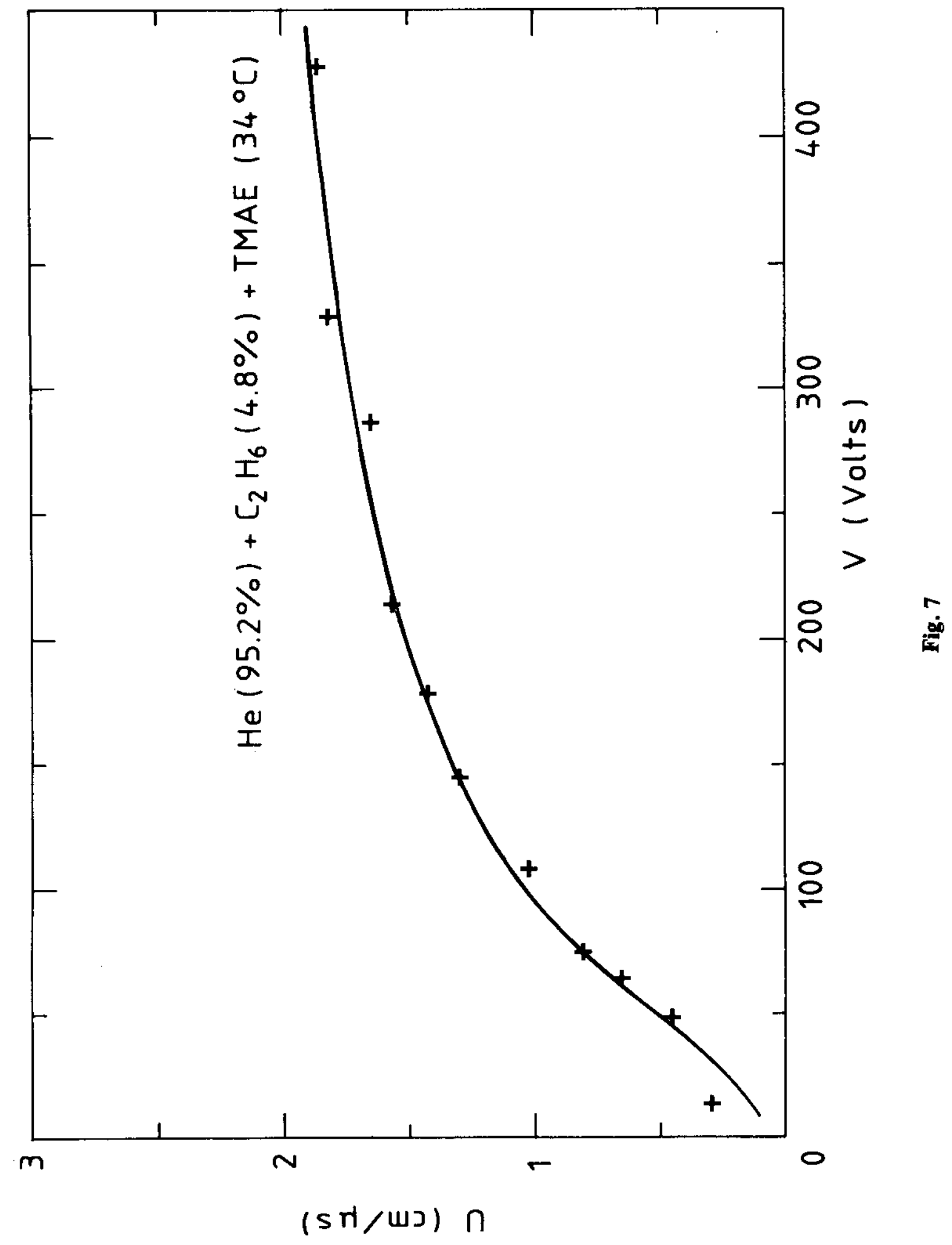




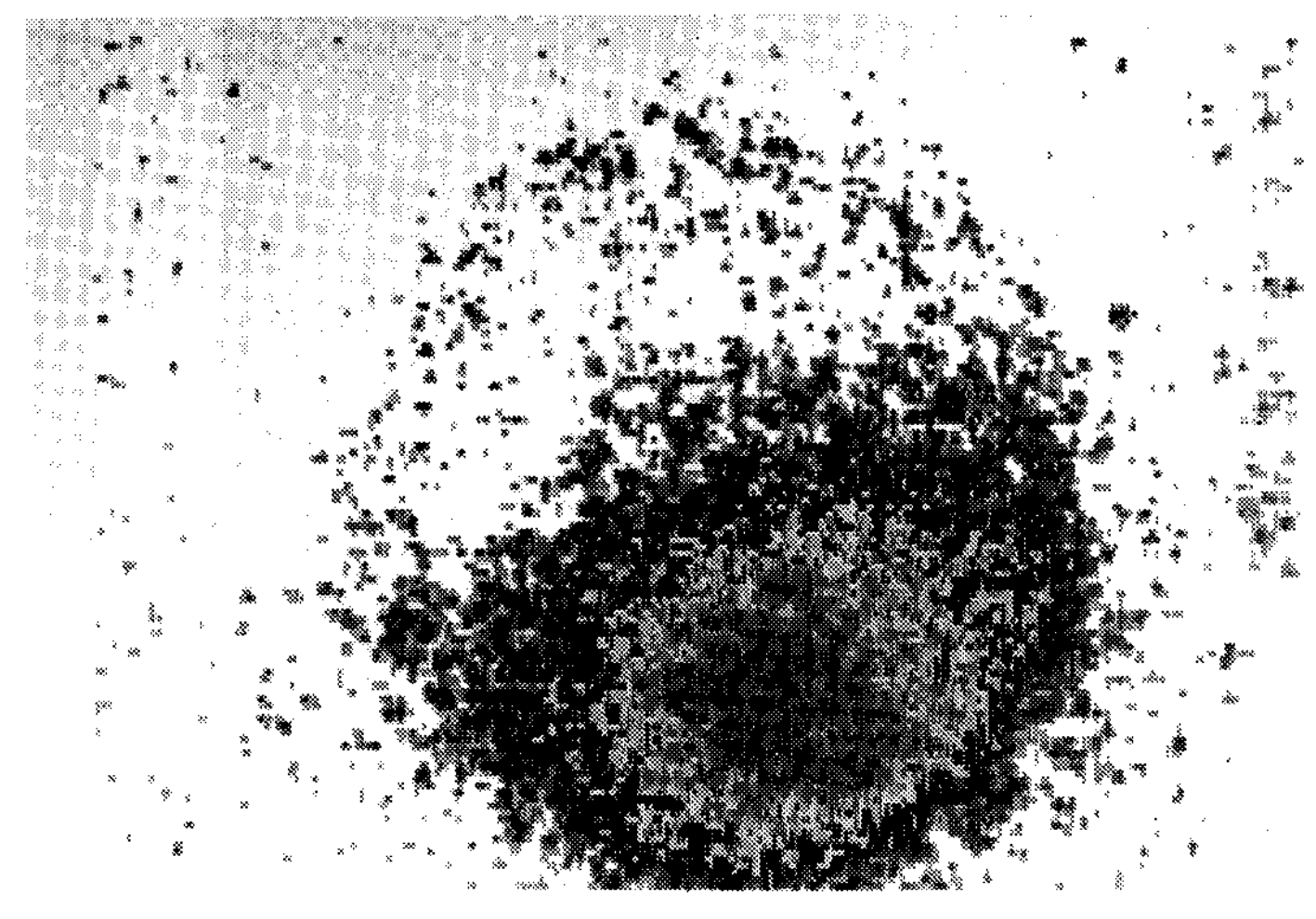

Fig. 8

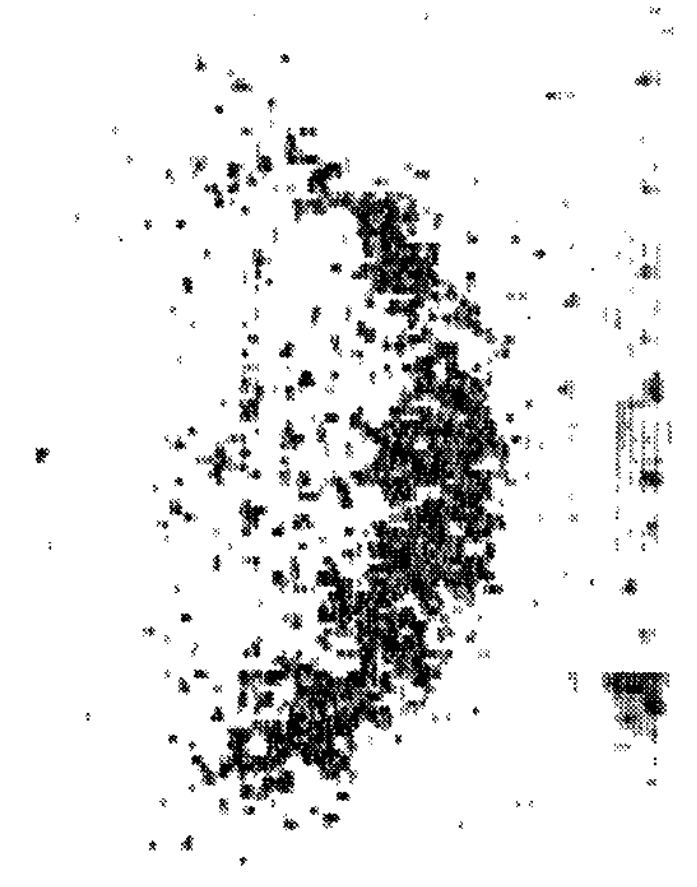

Fig. 9 


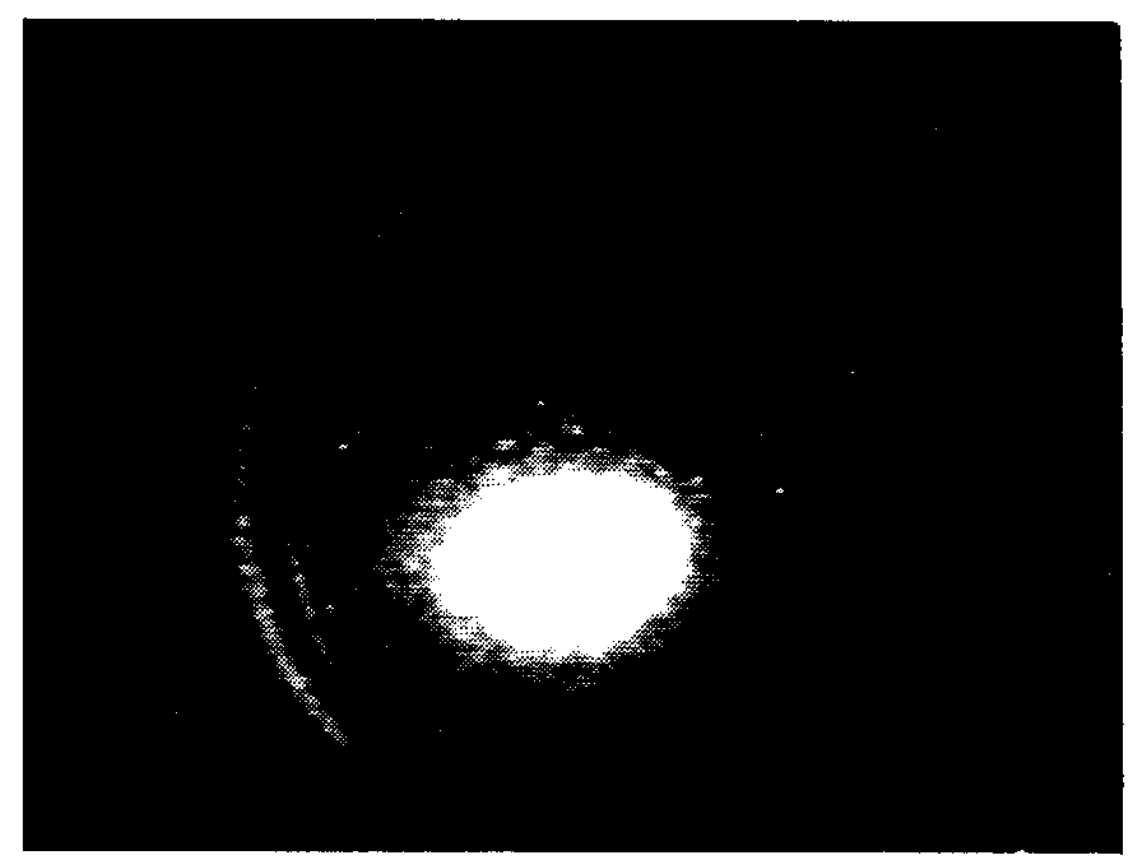

Fig. 10

\section{RADIATOR}

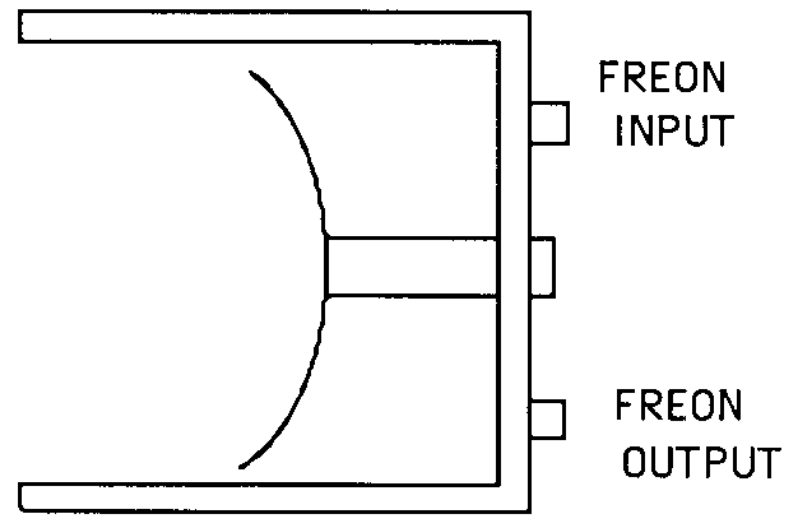

Fig. 11 


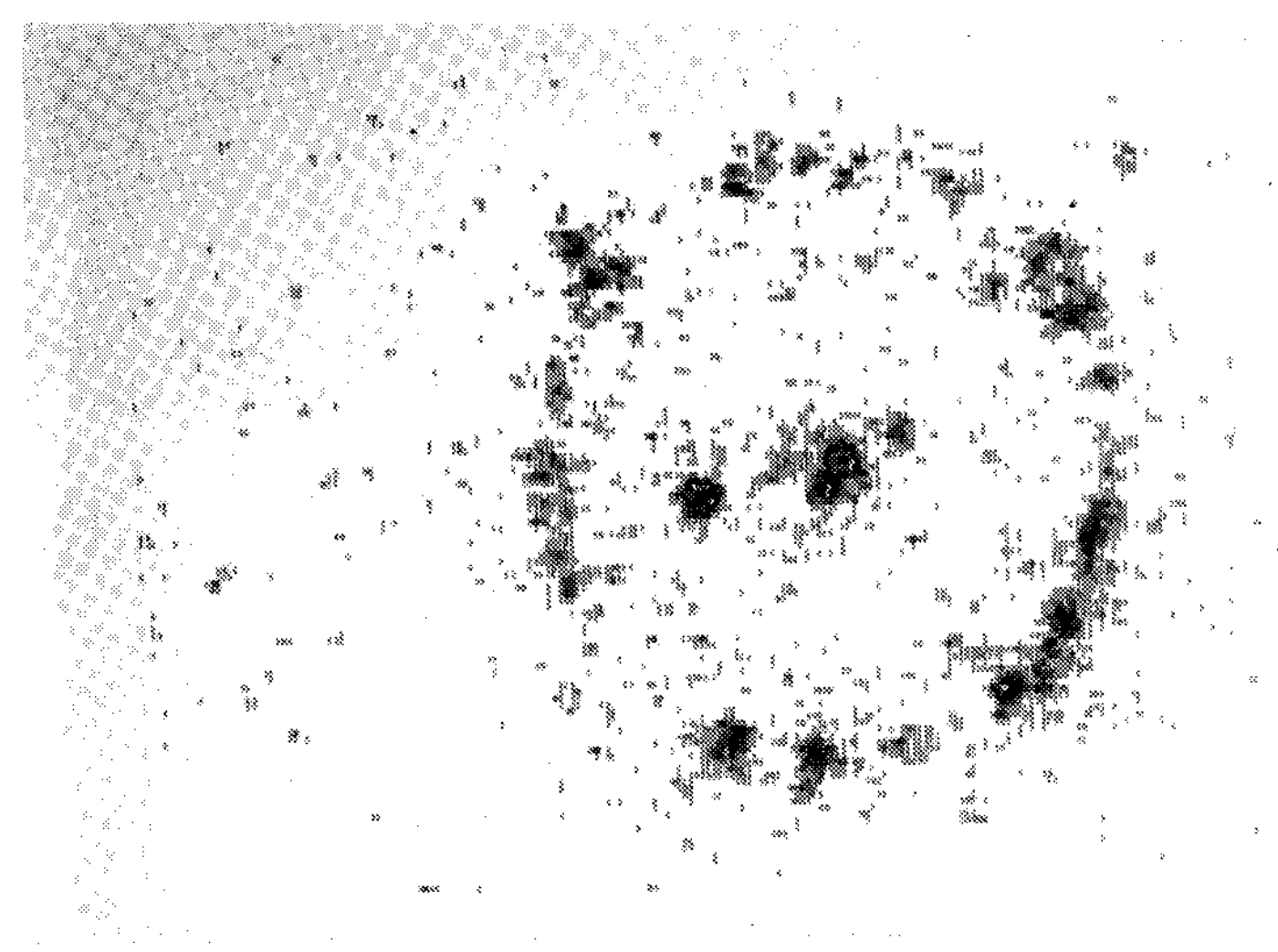

Fig. 12

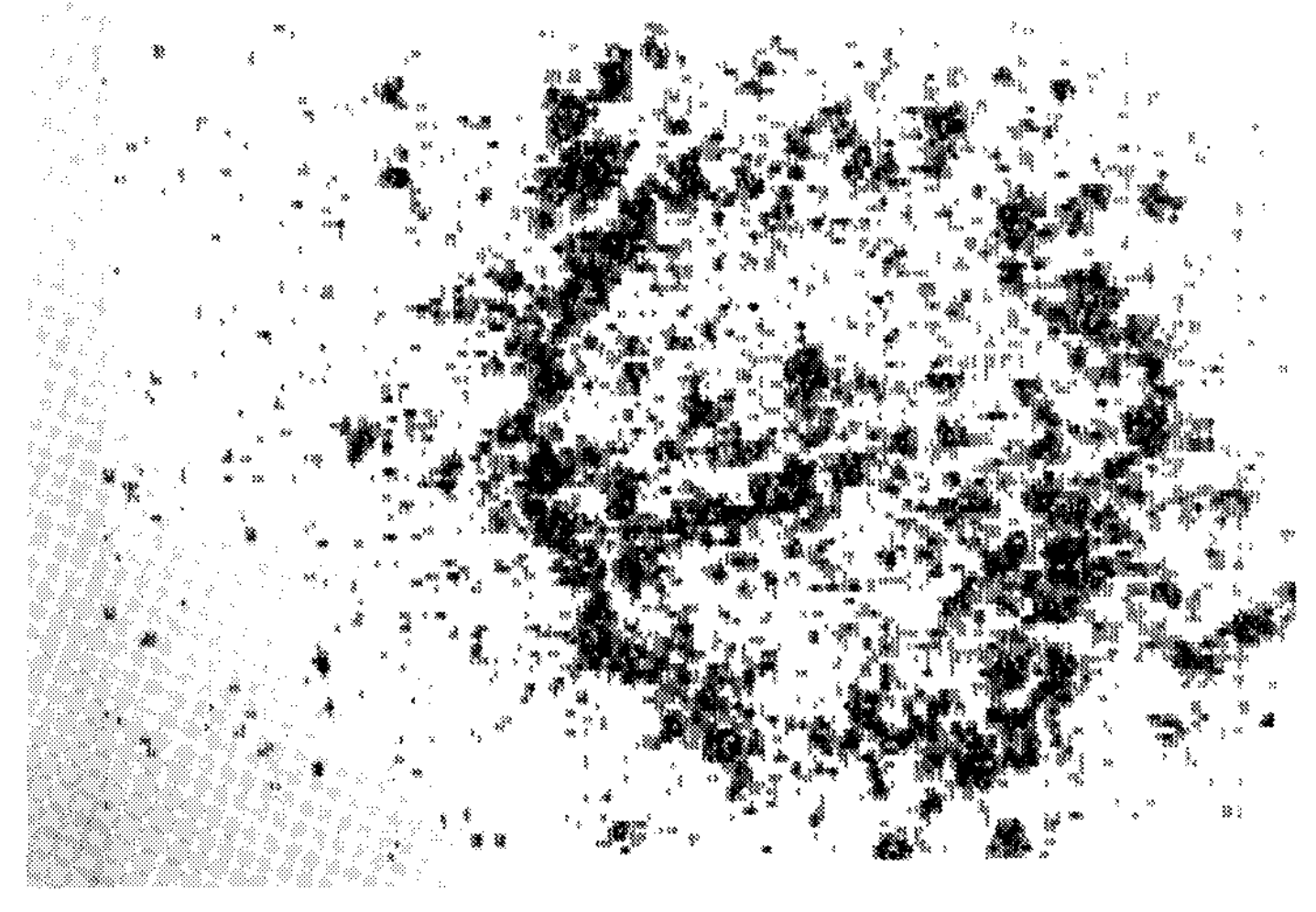

Fig. 13 